\title{
WAVANGLET: An Efficient Supervised Classifier for Hyperspectral Images
}

\author{
Frédéric Schmidt, Sylvain Douté, and Bernard Schmitt
}

\begin{abstract}
The new generation of imaging spectrometers onboard planetary missions usually produce hundreds to thousands of images a year, each made up of a thousand to a million spectra with typically several hundred wavelengths. Such huge datasets must be analyzed by efficient yet accurate algorithms. A supervised automatic classification method (hereafter called "wavanglet") is proposed to identify spectral features and classify images in spectrally homogeneous units. It uses four steps: 1) selection of a library composed of reference spectra; 2) application of a Daubechies wavelet transform to referenced spectra and determination of the wavelet subspace that best separates all referenced spectra; and 3) in this selected subspace, determination of the best threshold on the spectral angle to produce detection masks. This application is focused on the Martian polar regions that present three main types of terrains: $\mathrm{H}_{2} \mathrm{O}$ ice, $\mathrm{CO}_{2}$ ice, and dust. The wavanglet method is implemented to detect these major compounds on near-infrared hyperspectral images acquired by the OMEGA instrument onboard the Mars Express spacecraft. With an overall accuracy of $89 \%$, wavanglet outperforms two generic methods: band ratio ( $57 \%$ accuracy) and spectral feature fitting $(83 \%$ accuracy). The quantitative detection limits of wavanglet are also evaluated in terms of abundance for $\mathrm{H}_{2} \mathrm{O}$ and $\mathrm{CO}_{2}$ ices in order to improve the interpretation of the masks.
\end{abstract}

Index Terms-Automatic detection, automatic supervised classification, hyperspectral images, mass treatment, pattern recognition, remote sensing, spectral feature recognition and extraction, wavelet filtering.

\section{INTRODUCTION}

$\mathbf{I}$ $\mathrm{N}$ ORDER to understand the current climate on Mars, it is necessary to detect, characterize, and monitor $\mathrm{CO}_{2}$ and $\mathrm{H}_{2} \mathrm{O}$ ices at its surface (permanent and seasonal deposits) and in its atmosphere (vapor, clouds). The series of images acquired above medium latitudes and the poles by the OMEGA [1] imaging spectrometer onboard the Mars Express (MEX) mission [European Space Agency (ESA)] represents a unique opportunity to achieve these objectives. Currently, it contains more than a hundred visible and near-infrared hyperspectral images taken during different seasons above the north and south polar regions with usually $\sim 100000$ spectra for each image.

Manuscript received May 12, 2006; revised September 23, 2006. This work was supported in part by a Ph.D. Grant from the "Ministère délégué à l'Enseignement supérieur et à la Recherche" and in part by a contract with CNES through its "Groupe Système Solaire" programs.

The authors are with the Laboratoire de Planétologie de GrenobleCNRS/UJF, Bâtiment D de Physique, 38041 Grenoble Cedex 09, France.

Color versions of one or more of the figures in this paper are available online at http://ieeexplore.iee.org.

Digital Object Identifier 10.1109/TGRS.2006.890577
We aim to do the following:

1) evaluate the relevance of each image for volatile molecule studies in terms of fractions covered by $\mathrm{CO}_{2}$ and $\mathrm{H}_{2} \mathrm{O}$ ices;

2) generate $\mathrm{CO}_{2}$ and $\mathrm{H}_{2} \mathrm{O}$ ice distribution maps;

3) automatically determine the seasonal condensation and defrost lines and their temporal evolution;

4) define spectrally homogeneous units of terrain as detection masks, each undergoing a specific physical modeling of the spectra in order to evaluate their surface properties.

Because of the huge volume of data, we need an automatic and efficient algorithm to achieve these goals in a reasonably short calculation time. In supervised classification, the usual way to compare each spectra $X$ of a hyperspectral image and a well-known reference spectrum $E$ is to calculate their spectral angle (SA) [2] in the $n$-dimensional base $(n=$ the number of channels) which is equivalent to the correlation coefficient. However, this method is not robust enough to classify the polar terrains because of the slightly degraded signal-to-noise ratio of the spectra mostly acquired at high incidence angles. Furthermore, gaseous $\mathrm{CO}_{2}$ and $\mathrm{H}_{2} \mathrm{O}$ present in the atmosphere add absorption features that sometimes partly overlap their solid counterparts. Finally, variations of the physical properties of the ices (e.g., grain size) and variations of geometrical illumination conditions induce nonlinear variations of the absorption band intensity across the studied scene that are impossible to unravel using a linear SA method. Therefore, we propose a supervised classification method called wavanglet. It defines a more effective way of measuring the SA between the image spectrum $X$ and the reference spectrum $E$ in a wavelet transform (WT) domain. The reference is usually an image endmember or a synthetic spectrum that nonambiguously characterizes the compound to be detected, e.g., $\mathrm{CO}_{2}$ or $\mathrm{H}_{2} \mathrm{O}$ ice owing to their distinctive absorption bands. Similar methods have already been evaluated in remote sensing. The use of wavelets for pattern recognition was already proposed by [3] and [4], and their efficiency is better than that provided by other techniques such as principal component analysis (PCA) and Fourier transforms [5]. Experimental studies have shown that the performance of wavelets in classification is very good for vegetation detection with Airborne Visible/Infrared Imaging Spectrometer data [6] and hyperspectral data collected at ground level [7].

The use of unsupervised classification methods like PCA [8] or hierarchical clustering [9] is limited when processing large 
TABLE I

Basic SPECTRAL CHARACTERISTICS OF THE OMEGA INSTRUMENT ONBOARD MEX

\begin{tabular}{|c|c|c|c|}
\hline & V channel & C channel & L channel \\
\hline \hline Spectral Range $(\mu \mathrm{m})$ & 0.38 to 1.05 & 0.93 to 2.73 & 2.55 to 5.1 \\
\hline Number of Samples & 96 or 144 & 128 & 128 \\
\hline Spectral Resolution $(\mu \mathrm{m})$ & 0.007 or 0.0045 & 0.013 & 0.020 \\
\hline
\end{tabular}

datasets because such methods are computer intensive. Furthermore, they do not take advantage of the a priori knowledge we may have concerning planetary surfaces and atmospheres. Finally, they classify an image according to multiple chemical, physical, and structural information that are combined. Thus, the classification can be difficult to interpret in terms of chemical composition only, a problem that we do not encounter with wavanglet.

This paper is divided into four sections. In the first, we provide background information necessary to introduce the wavanglet method and the OMEGA dataset on which we will base our classification experiments. We also describe comparable standard methods that already exist: band ratio (BR) [10], [11], Spectral Angle Mapper (SAM) [2], and spectral feature fitting (SFF) [12]. The second section presents the different steps followed by our method. Next, we will perform a comparative experiment between wavanglet and two standard methods based on performance (overall accuracy, separability between classes, multiple endmembers and possible overlapping signatures, mass processing feasibility, and calculation time). Finally, in the particular case of the OMEGA polar images, we evaluate quantitative detection limits in terms of abundance for $\mathrm{H}_{2} \mathrm{O}$ and $\mathrm{CO}_{2}$ ices. We also determine how these limits vary with the coexistence mode of the ices, their main physical parameters (e.g., granularity) and the incidence angle of the observation. For this, we use synthetic data.

\section{BACKGROUND INFORMATION}

\section{A. OMEGA Instrument}

The OMEGA instrument [1] is an imaging spectrometer onboard the MEX-ESA. Its spatial resolution varies from $350 \mathrm{~m}$ up to $4 \mathrm{~km}$ depending on the observation altitude. The instrument has three distinct spectral channels designated V, C, and $\mathrm{L}$. Table I summarizes the spectral range, number of spectels, and spectral resolution for each. Unlike the $\mathrm{V}$ channel, the $\mathrm{C}$ and $\mathrm{L}$ channels are particularly relevant to our studies since they sample numerous absorption bands distinctive of $\mathrm{CO}_{2}$ and $\mathrm{H}_{2} \mathrm{O}$ in their solid state. After calibration, the dimensionless physical unit used to express the spectra is the reflectance: the irradiance leaving each pixel toward the sensor divided by the solar irradiance at the ground. Each pixel is characterized by a spectrum of 256 spectels organized according to increasing wavelength, the corresponding index going from 0 to 255 . The name of each observation is coded as ORBXXXX_Y where $\mathrm{XXXX}$ is the orbit number and $\mathrm{Y}$ the observation number.

\section{B. Wavelet Transform}

Our signals are modeled by finite energy functions of the $\mathbf{H}$ Hilbert space with the usual norm of the $L^{2}(\mathbb{R})$ Banach space and the usual inner product $\langle;\rangle$. As the Fourier transform is a projection on an orthogonal base made of orthogonal sine and cosine functions, the WT is a projection into a new base made of orthogonal wavelets. Each wavelet is built by scaling and shifting a single "mother" wavelet [13]. Consequently, a wavelet is defined by a mother pattern, a scale " $s$," and a position " $p$."

The mother wavelet is as follows:

1) a function

$$
\Psi \in L^{2}(\mathbb{R})
$$

2) with a zero average

$$
\int_{-\infty}^{+\infty} \Psi(x) d x=0
$$

3) centered in the neighborhood of $t=0$

4) normalized such that

$$
\|\Psi\|=1 .
$$

It is used to generate a family of functions: $\Psi_{s, p}(x)=$ $(1 / \sqrt{s}) \Psi((x-p) / s)$

1) which could be a base of $L^{2}(\mathbb{R})$;

2) which could have the orthonormal property: $\forall\left(s, p, s^{\prime}, p^{\prime}\right)$, $\left\langle\Psi_{s, p} ; \Psi_{s^{\prime}, p^{\prime}}\right\rangle=\delta_{s, s^{\prime}} \cdot \delta_{p, p^{\prime}}$.

The originality of wavelets compared to the usual sine and cosine functions is that each base function is localized. A cosine function, instead, is defined by its period and its phase, and is present throughout the entire signal.

The WT of the signal-vector $E(x)$ is:

$$
\mathrm{WE}(s, p)=\left\langle E, \Psi_{s, p}\right\rangle=\int_{-\infty}^{+\infty} E(x) \frac{1}{\sqrt{s}} \Psi^{*}\left(\frac{x-p}{s}\right) d x .
$$

This transformation is linear and can be written by the usual convolution product:

$$
\mathrm{WE}(s, p)=\int_{-\infty}^{+\infty} E(x) \frac{1}{\sqrt{s}} \Psi^{*}\left(\frac{x-p}{s}\right) d x=f \star \Psi_{s}
$$

with $\Psi_{s}=(1 / \sqrt{s}) \Psi^{*}(-x / s)$.

In signal processing, several classes of mother wavelets exist, each with different properties (including nonbase and nonorthogonality). We build our wavanglet method on an orthonormal base made using the Daubechies wavelet family. The corresponding transform algorithm was specially developed for fast numerical calculation [14]. Faster than the usual WTs [4], this particular WT is particularly suitable for the detection and classification of spectral features on large datasets. The morphology of the Daubechies mother wavelet can be tuned by a parameter-with 4,16 , or 20 as possible values-that depends on the regularity of the signal. If the latter is undersampled, the regularity is low and the best suited mother wavelet must be highly irregular and as short as possible (value 4). If sampling 


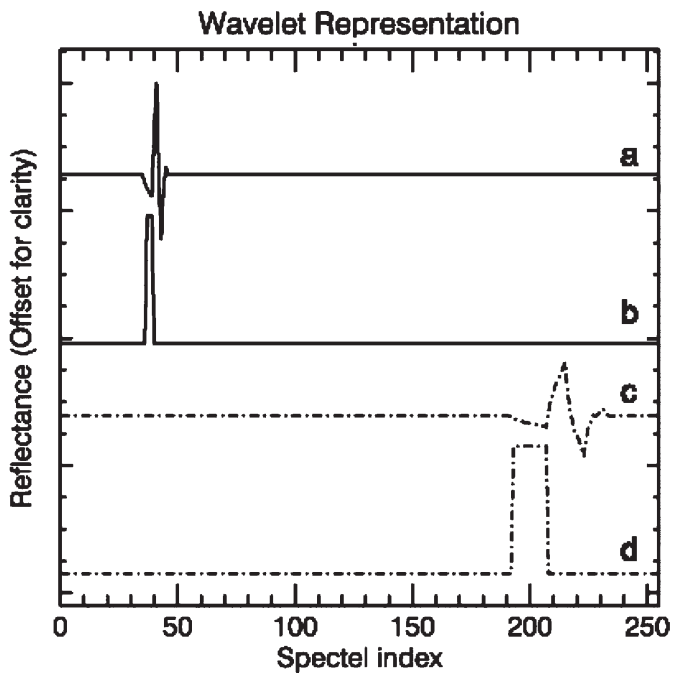

Fig. 1. (a) Daubechies wavelet representation. Wavelet No 28 (Scale 5 Position 13) and (b) its representation. (c) Wavelet No 73 (Scale 7 Position 10) and (d) its representation.

is sufficient, the mother wavelet must be smooth and long (value 20). The OMEGA data correspond to the first case. The discrete WT algorithm transforms a $2^{k}$ element vector into a vector of the same length. The counterpart of the spectel index in the wavelet domain will be called the wavelet index. The length $2^{k}$ of the vector determines the number of scales $k, 8$ for OMEGA, due to a spectral dimension of 256. The scale $s$ and position $p$ are not explicitly specified in the output vector but can be easily reconstructed. Each wavelet index corresponds to a unique wavelet with scale $s$ and position $p$. Different representations of the vector can be used in the wavelet space: for instance the primary output vector or a scale/position scheme. We will use a convenient representation with each scale represented by a $2^{k}$ element vector resulting from the superposition of gate functions, one for each wavelet, with a width equal to the scale $s$, a localization equal to position $p$, and a depth equal to the projection coefficient (see Fig. 1).

WTs can be used in spectral pattern recognition as proposed several years ago [3], [4]. The idea is that the wavelet localization and scale, respectively, correspond to the position and width of a particular spectral feature. Furthermore, the projection coefficient is linked to feature depth. In the case of a hyperspectral planetary image, each absorption band can be represented by the sum of a limited number of wavelets. In an ideal case, only the projection coefficients for this group of wavelets would differ from zero.

\section{Usual Classification Methods}

Many different methods of detection and classification are used in the domain of planetary sciences. We will only summarize three among the most popular.

1) BR Method: A ratio between two channels of interest can be calculated for all the spectra of an image. For instance, one channel can be chosen in the middle of an absorption band and the other on its far wing (local continuum). In this case, the ratio is straightforwardly linked to the relative band depth. a detection mask can be computed by applying a fixed
TABLE II

WAVELENGTHS USED IN THE BR METHOD

\begin{tabular}{|c|c|c|c|c|}
\hline Spectel index & 35 & 40 & 60 & 75 \\
\hline \hline Wavelength $(\mu m)$ & 1.4286 & 1.5004 & 1.7860 & 1.9973 \\
\hline
\end{tabular}

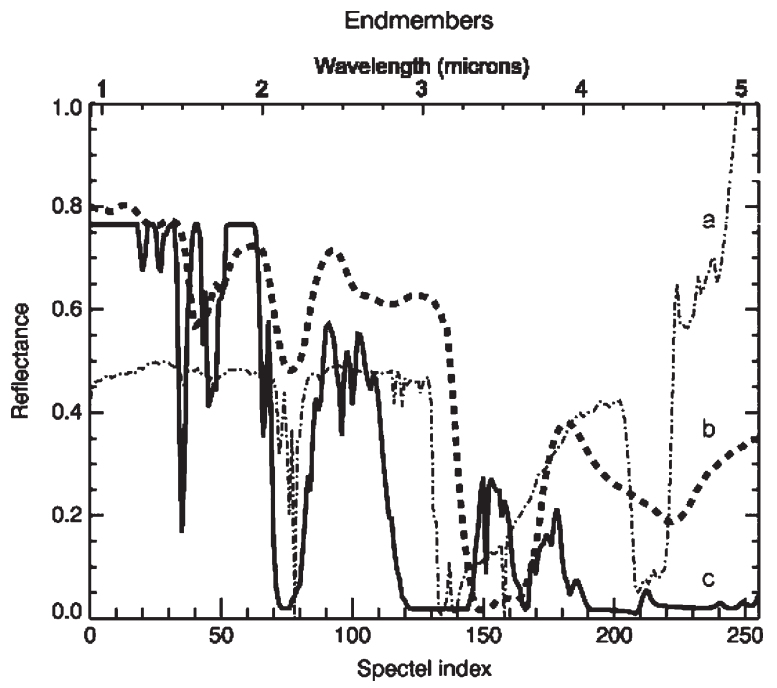

Fig. 2. Spectral endmembers. (a) Dust + atmosphere spectrum, (b) synthetic spectrum of $\mathrm{H}_{2} \mathrm{O}$ ice, and (c) synthetic spectrum of $\mathrm{CO}_{2}$ ice.

threshold on the image ratio, above which we consider that the band exists. The threshold can be calibrated by a visual inspection of representative spectra. Sometimes the definition of the BR is slightly more refined, e.g., the area sustained by the absorption band divided by its continuum. The main advantage of the ratio method is its simplicity. Nevertheless, this method is only used for preliminary investigations because of a number of limitations: dependence of hot spectels and sensitivity to nonmultiplicative noise, instrumental artifacts, and frequent superposition of different absorption bands. This method has already been applied to MEX OMEGA data [10], [11].

The BR we used for our classification experiments (see Section IV-B) is $(S(40) / S(35))(1-(S(75) / S(60)))$ with the associated wavelengths listed in Table II. Spectel index 35 corresponds to a $\mathrm{CO}_{2}$ solid absorption band and spectel index 40 to a water ice absorption. Spectel index 60 is the continuum, and spectel index 75 corresponds to both $\mathrm{CO}_{2}$ and $\mathrm{H}_{2} \mathrm{O}$ absorption bands (see Fig. 2 for the reference spectra). Terrains with a minimum value of the ratio are composed of dust. $\mathrm{CO}_{2}$-icerich terrains are represented by pixels with a high ratio. In between, water-ice-rich terrains have intermediate values (see Fig. 4 for the BR distribution in one OMEGA observation). An overview of the properties of this method and the results from experiments are reported (later) in Table IX.

2) $S A$ : A collection of spectra produced by a given instrument can be represented by a series of vectors each anchored at the origin of an $n$-dimensional space ( $n$ being the number of channels of the instrument). In a usual 3-D Euclidean space, the SA corresponds to the real angle between the two directions defined by two vectors [2]. In a general $n-d$ space, the SA is a coefficient $\alpha$ between 0 and $\pi$ radians which determines the proximity of the directions of two vectors $E$ and $X$. 
The SA:

$$
\alpha(E ; X)=\arccos \left(\frac{\langle E ; X\rangle}{\|E\| \cdot\|X\|)}\right)=\arccos (\operatorname{cor}(E ; X))
$$

The SA is not sensitive to a multiplication factor because it is normalized. This method is often used in remote sensing analysis to compare two spectra (vectors) because the photometric effect is not taken into account. Another way to introduce the $\mathrm{SA}$ is to present it as a correlation coefficient between two random vectors (the spectra) combined with an arc-cosine. As the arc-cosine is a monotonically declining function in the $[0,1]$ interval, the SA decreases as the normalized correlation increases. In the extreme case, when the two directions are similar, SA tends to 0 and the normalized correlation tends to 1 . When the two vectors are anticorrelated, the correlation coefficient is close to -1 , and thus the SA is close to $\pi$ radians. The intermediary situation is when the angle is $\pi / 2$ and the correlation is null.

The value of the SA does not depend on the base used for the vector representation if the new base is orthogonal for the usual scalar product. This means that if the scalar product between two vectors is preserved, the SA is also preserved.

If $\Phi$ is a self-adjoint linear operator such as WT, e.g., the projection into an orthonormal base of the $\mathbf{H}$ Hilbert space, we can write the following:

The Parseval formula

$$
\langle\Phi(E) ; \Phi(X)\rangle=\langle E ; X\rangle
$$

and the Plancherel formula

$$
\|\Phi(X)\|=\langle\Phi(X) ; \Phi(X)\rangle=\langle X ; X\rangle=\|X\|
$$

and thus

$$
\begin{aligned}
& \alpha\{\Phi(E) ; \Phi(X)\}=\arccos \left(\frac{\langle\Phi(E) ; \Phi(X)\rangle}{\|\Phi(E)\| \cdot\|\Phi(X)\|}\right) \\
& \alpha\{\Phi(E) ; \Phi(X)\}=\arccos \left(\frac{\langle E ; X\rangle}{\|E\| \cdot\|X\|}\right) \\
& \alpha\{\Phi(E) ; \Phi(X)\}=\alpha(E ; X) .
\end{aligned}
$$

SA is an algorithm already implemented in the environment for visualizing images (ENVI) environment [15].

3) Spectral Feature Fitting: SFF is a method that compares the spectrum of an image $X$ to a selected reference spectrum $E$ in a least-squares sense [12]. The reference spectrum is scaled to match the image spectrum after continuum removal for both spectra and the root mean square (rms) error is determined. We choose to express the similarity between $X$ and $E$ by calculating the combined ratio: scale/rms. The scale and the rms are, respectively, an estimation of the strength of the searched absorption features and of the quality of the fit. A simple threshold can be applied to the ratio to decide if the observed spectrum belongs to the class of the reference spectrum or not.

An overview of the properties of this method and the results from experiments are also reported in Table IX. SFF is also an algorithm included in the ENVI environment [15].

\section{Proposed Method: Wavanglet, SA Classification in a Wavelet Filtered Space}

The proposed wavanglet method is a succession of four steps. Step A is the determination of significant reference spectra $E$ characteristic of the different types of terrains present in the scene (the set is called the "reference base"). Step B is the choice of the most discriminating wavelet subspace for SA classification of the observed spectra $X$. Step $\mathrm{C}$ is the determination of the best threshold for the SA to produce detection masks ( $0=$ nondetection; $1=$ detection $)$. Step D is the automatic mass classification for the complete dataset.

\section{A. Step A: Choice of Relevant Reference Spectra}

This first step is crucial in the process because it will strongly affect the validity of the detection. If some species are not represented in the reference base, they will be misclassified in the dataset. If other species are represented in the base but are not present in the observation, the classification will not be optimized. This method is supervised because the a priori knowledge used to perform the classification is strong: number of present compounds in the image, nature of the compounds, etc. The user must supply a reference base that can be a set of endmembers extracted from the hyperspectral images and/or a set of synthetic spectra generated by a physical model. Endmembers can be extracted manually or automatically, for instance by a pixel purity index routine [16], after a dimension reduction performed by different techniques such as PCA or minimum noise fraction [17]. The synthetic spectra can be calculated with a bidirectional reflectance model adapted to the type of planetary surfaces observed [18]. A single chemical species with homogeneous physical properties (grain size, roughness, etc.) or a geographical/granular mixture of different chemical species with different surface properties may be used under certain circumstances. We will denote the $i$ th spectra of the reference base as $E_{i}$. Our algorithm will detect a reference spectrum $E_{i}$ in an observed spectrum $X$ when the signatures of the former are found in the latter with similar position, width, and relative depth. However, this method will neither be sensitive to the general level of the spectra nor to the absolute depth of bands for a particular species.

\section{B. Step B: Determination of the Best Subspace}

Several methods have been proposed to reduce the dimension, such as band selection on a well-documented learning dataset using discriminant analysis [19], or directly on the hyperspectral image avoiding correlation [20]. We will use the discrete WT, which is already used to increase the classification accuracy [21], in a alternative way.

1) Step B1-Continuum Removal: Real observed spectra commonly result from the superposition of multiple absorption bands due to several pure compounds mixed at different spatial scales. Such absorption bands undergo nonlinear variations when the physical properties of the materials present in the scene change [22]. Moreover, these bands appear on a continuum that is highly variable due to changes of illumination and global albedo. These are the main limiting effects when 
comparing the observed spectra with the image or synthetic endmembers. In order to reduce the sensitivity of our algorithm to such variations - which cannot be taken into account by our fixed reference endmembers-we perform the comparison only at medium and small wavelet scales $(s \in[5-8]$ for OMEGA classification). In this first filtered space, all wavelet coefficients mainly depend on the absorption band positions, widths, and relative depths. They are only moderately affected by noise in the spectra as shown by the experiments we carried out (see Section IV-C). One specific absorption band is usually represented by several wavelets.

2) Step B2-Best Discrimination: Additionally, we have to select the ideal subspace that allows the best discrimination among the different endmembers. It is possible to separate overlapping signatures during the decomposition process if they have different positions or widths, i.e., if we can find one wavelet at medium or small scale, specific to one absorption band but not polluted by another absorption band.

Qualitatively speaking, this subspace is composed of wavelets on which the reference spectra show the greatest differences. We propose three simple methods to carry out the selection.

$\mathrm{WE}_{i}(s, p)$ denotes the projection of the $i$ th spectrum of the reference base on the wavelet $\Psi_{s, p}$ at scale $s$ and position $p$.

1) Threshold for only one single reference spectra in the base:

Keep $\Psi_{s, p}$ if $\exists i$ such that

$$
\left\|\mathrm{WE}_{i}(s, p)\right\|>\operatorname{thres}(s) .
$$

2) Threshold for an extended spectral base:

Keep $\Psi_{s, p}$ if $\exists(i, j)$ such that

$$
\left\|\mathrm{WE}_{i}(s, p)-\mathrm{WE}_{j}(s, p)\right\|>\operatorname{thres}(s, i, j)
$$

with

$$
\text { thres }(s, i, j)=\text { const. }
$$

3) Automatic threshold method, same as method 2) above with

$$
\begin{array}{r}
\operatorname{thres}(s, i, j)=\operatorname{mean}_{i, j}\left\{\left\|\mathrm{WE}_{i}(s, p)-\mathrm{WE}_{j}(s, p)\right\|\right\} \\
+c * \operatorname{std}_{i, j}\left\{\left\|\mathrm{WE}_{i}(s, p)-\mathrm{WE}_{j}(s, p)\right\|\right\}
\end{array}
$$

Both mean $\operatorname{mean}_{i, j}$ and standard deviation $\operatorname{std}_{i, j}$ are calculated for combinations of $i$ and $j$ for a given $s$. The coefficient $c$ is determined by the user.

The norm \|| || can be $L^{1}, L^{2}$, or any other norm definition. We choose $L^{2}$ to be consistent with previous considerations concerning WTs in a $\mathbf{H}$ Hilbert space (see Section II-B).

Methods 1) and 2) cannot easily be carried out if the number of reference spectra is high because the number of thresholds becomes excessive. An alternative is method 3) which automatically determines the threshold. Other more complicated automatic methods have already been proposed in the literature, for example selection of the maximum by scale instead of a threshold criterion [3] or the best basis algorithm [23], [24].
3) Step B3-Circularity, Noise, and Dead Channels: The first problem that arises when analyzing numerical data by a WT is the edge effect. The circularity of the sampling creates false information symmetrically before and after the measured signal. All wavelets near the edge are polluted by this construction. To solve such a problem, we eliminate all wavelets containing information inherited from the first or last instrument spectels. For the OMEGA images, this method is valid because no features of interest are located near the edge of the signal.

Also, wavelets corresponding to spectels affected by instrumental defects or noise can reduce the accuracy of the classification algorithm. These wavelets are determined by the following method. First, we built a null-vector with a length equal to the number of spectels in an OMEGA spectrum. Second, we change the value of the vector from zero to one only at the rank of a defective spectel in order to simulate the defect with a normalized energy of one. The base of the modeled defect is denoted $D_{i}$. Then, we perform a WT on this signal $D_{i}$ and eliminate all wavelets with a coefficient higher than a threshold, i.e., the wavelets most affected by the defect. If we choose a typical value of $D_{i}(s, p)>0.5$, we eliminate only the wavelets that receive more than $50 \%$ of the defect energy. This operation should be performed for all potential defects $i$.

\section{Step C: SA Thresholds}

The calculation of the SA between the reference spectrum $E$ and the observation spectrum $X$ gives the correlation coefficient between these two spectra in a determined space. The characteristics of an appropriate subspace are the following. The belonging wavelets must be corrected from continuum effect (Step B1), form a part of an absorption band and be discriminating for at least one reference spectrum (Step B2), and be almost free of noise and numerical problems (Step B3). The SA in this subspace can be interpreted as a measurement of the agreement between distinctive spectral features of a reference spectrum and those of an observed spectrum. A small value indicates a good match of spectral features whereas a value close to $\pi$ indicates total disagreement. Some analysis procedures we use subsequently in wavanglet require that we decide from the SA if a given compound, characterized by its associated reference spectrum, is present or absent in a pixel. In this case, after processing the whole image, we obtain detection masks for the compounds that indicate if a given treatment is to be applied or not for a given pixel.

To declare positive detection, the correlation coefficient must be higher than a certain threshold, or in an analogous manner, the SA must be lower than a limiting angle. Ideally, to build our detection algorithm, a unique limiting angle, one per compound, must be detected and valid for all observations. This limiting angle can be interpreted as a limiting condition adapting the mathematical problem to a physical one. Two methods can be performed to determine the series of thresholds: calibration using a controlled database of synthetic spectra-modeling approach [C1] - and/or an empirical approach based on an already analyzed subset of real data [C2]. The choice of the threshold must maximize the detection limit of each pure 
compound in the image, regardless of its physical properties, and and must minimize false detections.

As the SA calculation is invariant with any linear orthonormal transformation, such as the Daubechies WT, we have the choice to perform the SA mapping of all the pixels in the filtered wavelet base or in the filtered spectral space. The latter is reconstructed by the inverse transform of the filtered wavelet base. The calculation of the SA is faster in the filtered wavelet base than in the complete spectral space because the dimensions of the former are lower. We therefore choose to perform the calculation in the filtered wavelet base.

\section{Step D: Automatic Mass Classification With SAs in This Subspace}

All the parameters are now adjusted to perform the automated classification. For each image and each spectrum of the reference base, a detection mask will be created. For datasets spanning a long time range (several months to years), many parameters can vary. For instance, the bad channel list can change with time thus affecting Steps B2 and B3. If this happens, then Step C should be adapted according to the former steps.

\section{EXPERIMENTS}

We will now apply this general wavanglet method to a series of OMEGA/MEX hyperspectral images and compare the obtained classifications with the ones produced by two alternative methods: BR and SFF (Section II-C1 and -C3). We also evaluate quantitative detection limits for $\mathrm{H}_{2} \mathrm{O}$ and $\mathrm{CO}_{2}$ ices in terms of abundance. We use synthetic spectra for this. This paper consists of five tests: classification accuracy, separability between classes, multiple endmembers and possible overlapping signatures, mass processing feasibility, and calculation time.

\section{A. Application of the Wavanglet Method to the Omega Dataset}

We follow the four steps described in Section III to apply the method to a collection of OMEGA/MEX images covering the polar regions of Mars.

1) Step A-Choice of Relevant "Endmembers" (Reference Spectra): The OMEGA spectra display signatures characteristic of both the atmosphere and the surface. The atmospheric contribution is due to gaseous $\mathrm{CO}_{2}$ and, depending on weather conditions, to clouds of dust, $\mathrm{CO}_{2}$, and $\mathrm{H}_{2} \mathrm{O}$ ices. The spectral effect of the clouds can often be neglected for a first-order approximation. The surface contribution is due to a mixture of $\mathrm{H}_{2} \mathrm{O}$ ice, $\mathrm{CO}_{2}$ ice, and dust in various proportions. In the images, we try to detect the last three compounds to which we attribute reference spectra (see Fig. 2). Pure $\mathrm{H}_{2} \mathrm{O}$ and $\mathrm{CO}_{2}$ are represented by synthetic spectra computed by a reflectance model [18] using the physical parameters listed in Table III and an optical constant measured in the laboratory [25]-[27]. These parameters have been chosen to be compatible with recent studies of both south and north Martian polar regions [10], [11], [28], [29]. On the other hand, the third reference spectrum representing polar dust is extracted from a single OMEGA image covering the southern high latitudes by averaging all spectra within a relatively homogeneous region near $70^{\circ}$ longitude
TABLE III

SYNTHETIC ENDMEMBER PARAMETERS

\begin{tabular}{|c|c|c|}
\hline & pure $\mathrm{H}_{2} \mathrm{O}$ ice & pure $\mathrm{CO}_{2}$ ice \\
\hline \hline Grain size $(\mu m)$ & 10 & 100000 \\
\hline Incidence angle $\left(^{\circ}\right)$ & 85 & 85 \\
\hline Emergence Angle $\left(^{\circ}\right)$ & 0 & 0 \\
\hline
\end{tabular}

TABLE IV

LIST OF ELIMINATED WAVELETS

\begin{tabular}{|l|l|l|l|} 
Spectel indexes of dead spectels & 34 & 78 & 158
\end{tabular} \begin{tabular}{|l|l|l|l|}
\hline Wavelet indexes of eliminated wavelets & $35,71,144$ & 82,166 & 102,206 \\
\hline
\end{tabular}



Fig. 3. Representation of the selected subspace of (upper part) wavelets that best discriminate the endmembers (lower part). (a)-(d) Wavelets selected in scales 5 to 8. (e) Observed dust and atmosphere spectra, (f) synthetic $\mathrm{CO}_{2}$ ice, and $(\mathrm{g})$ synthetic $\mathrm{H}_{2} \mathrm{O}$ ice. See Section II-B for wavelet representation.

and $-77^{\circ}$ latitude. Note that this spectrum is almost featureless in the near-infrared range except for the $3-\mu \mathrm{m}$ band due to the hydration of the minerals and the absorption bands of atmospheric $\mathrm{CO}_{2}$. Globally, the dust spectrum does not display much spatial variation in this spectral range. Indeed, it is well mixed by winds and spread over wide areas. Thus, we can assume that our reference spectrum is representative of most areas of both polar regions. Our Martian studies focus mainly on the two first endmembers while the third endmember represents spectral features that appear in the data but which are not of interest to us.

2) Step B-Determination of the Best Subspace:

Step B1-Continuum removal: We use only the last four scales (from 5 to 8 ) in order to remove the contribution of the continuum.

Step B2-Best discrimination: We prefer the automatic threshold method [method 3)], with norm $L^{2}$ and the value $c=2.5$ to select the best subspace. This threshold criterion optimizes the classification.

Step B3-Circularity, noise, and dead spectels: We eliminate all wavelets containing a nonzero contribution from the last spectel number 255 .

We eliminate wavelets polluted by damaged spectels (number 34,78 , and 158) with an energy criterion $(D=0.45)$. Table IV lists the indexes of the eliminated wavelets.

Finally, the selected subspace is formed by the 12 wavelets shown in Fig. 3 and summarized in Table V. 
TABLE V

List OF SELECTED WAVELETS

\begin{tabular}{|c|c|}
\hline Scale & Indexes of selected wavelets \\
\hline \hline 5 & 28 \\
\hline 6 & 36,58 \\
\hline 7 & $73,80,95,118$ \\
\hline 8 & $145,148,149,160,161,163,164,165,175,192,202,239$ \\
\hline
\end{tabular}

3) Step C-Threshold Determination: We use two different procedures to constrain the SA threshold: numerical calibration [C1] and image calibration [C2]

C1-Numerical calibration: The idea is to generate a database of synthetic spectra that simulates the OMEGA dataset by varying the relative abundances of ices and dust as well as other physical parameters over realistic ranges of values. Then, we can apply the wavanglet method on this controlled data and adjust the angle threshold so as to maximize the detection sensitivity for $\mathrm{H}_{2} \mathrm{O}$ and $\mathrm{CO}_{2}$ ices while minimizing false detections.

The synthetic spectra are calculated by SPECTRIMAG, a bidirectional reflectance model that solves the radiative transfer of solar light through granular icy media [18]. The input data are the spectral optical constants of ice crystals and dust measured in the laboratory [25]-[27]. Each compound is also characterized by parameters such as grain size and porosity. A real OMEGA spectrum measures the surface reflectance of a geographical and/or granular mixture of $\mathrm{H}_{2} \mathrm{O}$ ice, $\mathrm{CO}_{2}$ ice, and dust at, or under, the pixel scale. In the first case, the resulting spectrum is a linear combination of the spectral reflectance signatures of the individual compounds. In the second case, the spectrum results from nonlinear physics aspects implemented in the model. In order to simulate as realistically as possible a Martian spectrum acquired by OMEGA, we also take into account the atmosphere and the instrumental noise contributions. The first contribution is introduced by multiplying each simulated spectrum by a constant atmospheric transmission spectrum calculated for an altitude of $-4800 \mathrm{~m}$ and typical Martian weather conditions by a line-by-line radiative transfer model [30]. We simulate the instrumental noise by a Gaussian process using statistics coming from dark current measurements of OMEGA observation 41_1 acquired during orbit 41.

C2-Image calibration: We carry out the training phase of the wavanglet and SFF methods using observation 41_1 that covers the south polar region during local summer $\left(\mathrm{Ls}=337.9^{\circ}\right)$.

A careful visual interpretation of observation 41_1 was carried out in order to select samples of pixels that we can define as "ground truth" regions of interest (GTROI). Dust, $\mathrm{H}_{2} \mathrm{O}$, and $\mathrm{CO}_{2}$ ices are identified on the basis of their distinct absorption bands. Nevertheless, this operation is very time consuming, and we need as many GTROIs as possible to carry out the training and subsequent testing (see Section IV-B) of the classification methods evaluated in this paper. Furthermore, the sensitivity of the brain/eye system is sometimes insufficient to recognize relevant spectral features in circumstances such as a very small amount of water ice mixed with solid $\mathrm{CO}_{2}$. As a consequence we use the limited $(\approx 30)$ manually obtained set of GTROIs for $41 \_1$ in order to train the BR method, which is always more sensitive than the human eye. We obtain two detection thresholds that, respectively, separate the $\mathrm{CO}_{2}$ class from the $\mathrm{H}_{2} \mathrm{O}$ class and the latter from the dust class. These thresholds are optimized for observation 41_1 or any other visually interpreted image. Then, we consider that the classification maps obtained with this optimized BR method (hereafter named BR_opt) constitute a reference for the training (observation 41_1) and testing (observation 231_1, see Section IV-B) of wavanglet as well as other classification methods.

Since our detection classes can overlap because both $\mathrm{CO}_{2}$ and $\mathrm{H}_{2} \mathrm{O}$ ices as well as dust can be detected in the same pixel, the training and testing are performed separately for the three compounds. In each case, we consider two classes: pixels with positive detection and pixels with negative detection. For a range of proposed SA thresholds (see Table VII), we calculate the overall accuracy and the kappa statistics [31] given by wavanglet by comparing its classification map with the reference map (BR_opt). The production and user accuracies are also calculated. We retain the threshold that maximizes the kappa factor. The training of SFF is performed in the same manner.

Both numerical and image calibrations converge on threshold angles of $1.54 \mathrm{rad}$ for $\mathrm{H}_{2} \mathrm{O}$ ice, $1.48 \mathrm{rad}$ for $\mathrm{CO}_{2}$ ice, and 0.34 for dust. Note that the threshold value is close to $\pi / 2$ for the ices whereas it is much lower for dust. Our reference spectra for $\mathrm{H}_{2} \mathrm{O}$ and $\mathrm{CO}_{2}$ do not contain any atmospheric contribution as opposed to the dust reference and to the images we classify. As a consequence, the observed spectra over icy terrains differ more from their reference spectra than those observed over the dusty terrains. In Section IV-C, we present the detection limits we achieve on the synthetic spectra in more detail.

4) Step D-Classification Using the Complete Dataset: The presentation and discussion of the results obtained by applying the wavanglet method to the complete OMEGA dataset are not the subject of this paper. We only discuss the ability of our algorithm to analyze a selection of representative observations. For that purpose, we carry out a comparative study with the BR, SAM, and SFF methods (see Section II-C).

\section{B. Comparison With Other Methods}

In this paper, we illustrate our tests with observation 231_1 of the north polar region recorded during local springtime (Mars solar longitude, $\mathrm{Ls}=8.6^{\circ}$ ). This observation presents different $\mathrm{H}_{2} \mathrm{O}$ and $\mathrm{CO}_{2}$ ice properties than observation 41_1, such as grain size and dust content [10], [11], [28].

All the results are summarized in Table IX.

1) Classification Accuracy: We admit that the optimized BR method (BR_opt for 231_1) is the best classification method and thus constitutes a reference (see Section IV-A3b). Yet, we cannot use it to classify the whole OMEGA dataset since it requires the manual constitution of GTROIs and subsequent manual training. As a consequence we only test and compare classification methods that could be used to process a large number of images automatically:

1) non-optimized BR method (hereafter called BR_fix);

2) SFF method;

3) "Wavanglet" method. 
TABLE VI

Classification AcCuracy for a Polar Observation (231_1) ACQuired By OMEGA. The Reference Method Is the BR Method Optimized

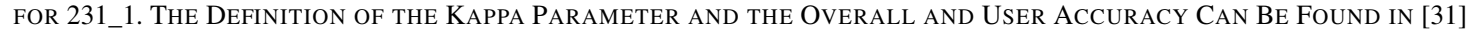

\begin{tabular}{|c|c|c|c|c||c|}
\hline Method & Threshold & Kappa & $\begin{array}{l}\text { Overall accuracy } \\
(\%)\end{array}$ & $\begin{array}{l}\text { user accuracy } \\
(\%) \text { detection }\end{array}$ & $\begin{array}{l}\text { user accuracy } \\
(\%) \text { no detection }\end{array}$ \\
\hline \hline BR_fix - $\mathrm{H}_{2} \mathrm{O}$ & $0.36-0.664$ & 0.071 & 35.6 & 16.8 & 97.0 \\
\hline SFFTM- $\mathrm{H}_{2} \mathrm{O}$ & 5.534 & 0.583 & 86.0 & 49.3 & 99.9 \\
\hline Wavanglet $-\mathrm{H}_{2} \mathrm{O}$ & 1.54 & 0.538 & 83.7 & 45.3 & 100.0 \\
\hline BR_fix - $\mathrm{CO}_{2}$ & 0.467 & 0.576 & 80.2 & 54.6 & 100.0 \\
\hline SFF & 3.716 & 0.572 & 80.0 & 54.4 & 99.9 \\
\hline Wavanglet $-\mathrm{CO}_{2}$ & 1.48 & 0.793 & 93.2 & 100 & 92.0 \\
\hline
\end{tabular}

All three methods use fixed detection thresholds to classify the dataset. For BR_fix, the thresholds equal those of BR_opt for observation 41_1.

Table VI shows the overall accuracy of the classifications achieved by wavanglet, SFF, and BR_fix using the optimized thresholds listed in Section IV-A3. In Table VI, note that for the detection of water ice terrains, SFF and wavanglet show similar detection capabilities with an overall accuracy of $86 \%$ and $84 \%$, respectively. On the other hand, BR_fix misclassifies $64 \%$ of the pixels spectrally dominated by $\mathrm{H}_{2} \mathrm{O}$. For $\mathrm{CO}_{2}$ terrains, wavanglet clearly outperforms SFF and BR_fix with an overall accuracy of $93 \%$ versus $80 \%$.

2) Separability Between Classes: Class separability must be considered to evaluate the robustness of a classification algorithm. In our case, each class corresponds to the positive detection of $\mathrm{H}_{2} \mathrm{O}$ ice, $\mathrm{CO}_{2}$ ice, and dust. First, we must consider a space in which the data can be represented. For wavanglet, this space is the SA in the wavelet filtered subspace, one for each endmember $E_{i}$. For the BR method, this space is the ratio, unique for all endmembers. In these representation spaces, a hyperspectral image is a collection of values, each representing one spectrum. The classification consists in defining a threshold in the distribution of SAs or a ratio for the whole population of spectra of a hyperspectral image. The distribution may have three types of groups: first with a dominant presence of the reference material corresponding to $E_{i}$ (group \#1), second with the presence of the same material mixed with other components (group \#2), third without the presence of the selected material corresponding to $E_{i}$ (group \#3). In the distribution, each group can be identified, and usually multiple peaks correspond to a single group. In our case, the intersection between materials are non-null: for instance, we can find materials with both $\mathrm{H}_{2} \mathrm{O}$ ice and $\mathrm{CO}_{2}$ ice (group \#2).

The less the intersection between group \#2 and group \#3 contains pixels, the more the classification will be reliable. One way to estimate the class separability is to evaluate the range of thresholds that give reasonably accurate detections. Robustness is ensured when the range of possible values is relatively wide compared to the width of the groups. This corresponds to a large distance between pixels from groups \#2 and \#3 in the representative space.

For the BR method (Fig. 4), the $\mathrm{H}_{2} \mathrm{O}, \mathrm{CO}_{2}$, and dust groups strongly overlap, and thus the range of possible thresholds is very limited. On the other hand, for the wavanglet method (Fig. 5), the SA distribution plots of the different materials, measured in their relative representing space, display wellseparated groups.

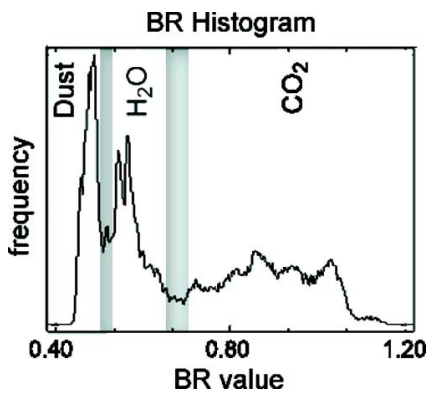

Fig. 4. BR value distribution for the 231_1 image. The gray boxes represent the range of thresholds possible for Dust $/ \mathrm{H}_{2} \mathrm{O}$ detection on the right and $\mathrm{H}_{2} \mathrm{O} / \mathrm{CO}_{2}$ detection on the left.


Fig. 5. SA distributions from the wavanglet method applied to the 231_1 image for (a) $\mathrm{H}_{2} \mathrm{O}$ ice, (b) $\mathrm{CO}_{2}$ ice, and (c) dust endmembers. The gray box represents the range of acceptable thresholds for reliable detection.

3) Multiple Endmembers and Possible Overlapping Signatures: When an increasing number of endmembers is required to classify a large dataset, it must still be possible to separate 
TABLE VII

Threshold Range of Positive Detection for the BR AND WaVANGlet Methods Based ON 41_1 ANd 231_1 OBservations

\begin{tabular}{|c|c|c|c|}
\hline Threshold & $\mathrm{H}_{2} \mathrm{O}$ ice & $\mathrm{CO}_{2}$ ice & dust \\
\hline \hline BR_opt on 41_1 & $<0.360(<0.398)$ to $>0.467(>0.664)$ & $>0.467$ to $>0.664$ & $<0.360$ to $<0.398$ \\
\hline BR_opt on 231_1 & $<0.507(<0.532)$ to $>0.651(>0.695)$ & $>0.651$ to $>0.695$ & $<0.507$ to $<0.532$ \\
\hline wavanglet opt. on 41_1 & $<1.5$ to $<1.56$ & $<1.4$ to $<1.53$ & $<0.3$ to $<0.6$ \\
\hline wavanglet opt. on 231_1 & $<1.45$ to $<1.57$ & $<1.43$ to $<1.52$ & $<0.32$ to $<0.58$ \\
\hline operational wavanglet & $<1.54$ & $<1.48$ & $<0.34$ \\
\hline
\end{tabular}

classes. The BR method, as presented above, does not work for the detection of more than a few species because it becomes impossible to find a ratio that ensures class separability, especially when the spectral signatures overlaps. On the other hand, the SFF and wavanglet methods are more suitable for the classification of large spectral datasets with multiple endmembers because they perform the classification based on an automatically defined spectral subspace. The user only needs to define the endmember collection.

4) Mass Processing Feasibility: Application of a classification method with a threshold (like wavanglet, BR, SFF) to a huge dataset requires a constant threshold from one image to the other. Table VII shows the acceptable range of thresholds for the BR and wavanglet methods based on observations 41_1 and 231_1. For BR, the threshold range between dust and $\mathrm{H}_{2} \mathrm{O}$ is strongly variable, and automation is therefore not possible. For the wavanglet method, the threshold range for a given compound varies only slightly, and a single constant value can be selected for optimized detection. The final thresholds determined in Section IV-A3 for $\mathrm{H}_{2} \mathrm{O}, \mathrm{CO}_{2}$, and dust are also reported in Table VII.

5) Calculation Time: We measured the computation time necessary to complete the classification of observation 41_1 (111616 spectra of 256 spectels) with the BR, SFF, and wavanglet methods. A complete process consists of opening files, running the classification algorithm, creating regions of interest, and saving product files. Each type of processing was performed in the ENVI environment on a LINUX workstation with the following characteristics: Intel Xeon $2.40-\mathrm{GHz}$ biprocessor with a 512-KB cache memory for each processor, hyperthreading active technology, 6 GB of RAM, OS Linux with kernel 2.6 SMP. Results of the tests are given in Table IX. Wavanglet is the slowest method, but its run-time is comparable to SFF.

\section{Evaluating Detection Limits With Synthetic Data}

We now evaluate the sensitivity of wavanglet for the detection of $\mathrm{H}_{2} \mathrm{O}$ and $\mathrm{CO}_{2}$ ices using synthetic data. For this, we consider that solid $\mathrm{H}_{2} \mathrm{O}, \mathrm{CO}_{2}$, and dust form a granular mixture with different mass proportions and grain sizes. For given observation conditions, we calculate the reflectance spectra that OMEGA would measure including the atmosphere contribution and the instrument noise (as explained in Section IV-A3-C1). We use different sets of parameters in the simulation. We span the whole range of mass proportions in a relatively continuous manner and use a limited set of values for the other parameters: atmospheric path, grain sizes, and incidence angle. The latter values, listed in Table VIII, are characteristics of polar ices [10], [11], [28], [29]. For each combination of parameters,
TABLE VIII

Set of Physical AND ObSERVATION PARAMETER VALUES USED TO GENERATE THE TERNARY COMPOSITION DiAgRAMS

\begin{tabular}{|c|c|c|c|}
\hline & Figure 6 and 7 & Figure 8 & Figure 9 \\
\hline \hline Grain size $\mathrm{H}_{2} \mathrm{O}$ ice $(\mu m)$ & 100 & 100 & $\mathbf{1 0 0 0}$ \\
\hline Grain size $\mathrm{CO}_{2}$ ice $(\mu m)$ & 45000 & 45000 & 45000 \\
\hline Grain size dust $(\mu m)$ & 30 & 30 & 30 \\
\hline Incidence angle $\left(^{\circ}\right)$ & 85 and 15 & 85 and 15 & 85 and 15 \\
\hline Emergence angle $\left(^{\circ}\right)$ & 0 & 0 & 0 \\
\hline Atmosphere & South & North (deeper) & South \\
\hline
\end{tabular}

we perform automatic detection of the ices using wavanglet. The reference base is composed of the three spectra described in Section IV-A1 (see Fig. 2). The results are represented in ternary diagrams with the three poles $\left(\mathrm{H}_{2} \mathrm{O}, \mathrm{CO}_{2}\right.$, and dust) at the edges of the triangle, one point inside the triangle corresponding to a triplet of mass proportions (their sum being always equal to unity). Each symbol represents a positive detection for a triplet. The absence of a symbol means no detection. At one triplet location two symbols can be superimposed on the triangular diagram: the inner and outer symbols correspond to incidence angles of $85^{\circ}$ and $15^{\circ}$, respectively. We can thus assess the effect of changing the atmospheric path, grain size, and incidence angle on the detection limits.

1) General Result: The detection limit for physical parameters of the typical surface (see Table VIII) of the permanent south polar cap is plotted in Fig. 6 and zoomed in Fig. 7. First, note that the general detection of $\mathrm{H}_{2} \mathrm{O}$ ice is good until near the dust corner and near the $\mathrm{CO}_{2}$ corner. Second, the detection of $\mathrm{CO}_{2}$ ice is only present near the pure $\mathrm{CO}_{2}$ ice pole. The difference between these two situations is related to the grain size effect. The grain size of $\mathrm{CO}_{2}$ ice is almost 500 times greater than the grain size of $\mathrm{H}_{2} \mathrm{O}$ ice and 1000 times greater than that of dust. In our case of granular mix, the mean free path is smaller inside $\mathrm{CO}_{2}$ ice than inside $\mathrm{H}_{2} \mathrm{O}$ ice or dust. The result is that even with a similar proportion of $\mathrm{H}_{2} \mathrm{O}, \mathrm{CO}_{2}$, and dust, the $\mathrm{CO}_{2}$ absorption bands will be smaller than the $\mathrm{H}_{2} \mathrm{O}$ and dust absorption bands. The presence of significant $\mathrm{CO}_{2}$ absorption bands in the spectra requires a high relative weight proportion of $\mathrm{CO}_{2}$ in order to balance this grain size effect.

2) Atmospheric Path: We generate two ternary diagrams for an atmosphere extending from space to an altitude of, respectively, $+4800 \mathrm{~m}$ (a situation typical of south polar region of Mars, Fig. 6) and $-4800 \mathrm{~m}$ (a situation typical of the northern lowland region, Fig. 8). We see in the figures that, despite a large atmosphere thickness difference, the atmospheric path has only a minor effect on our detection limits. The detection of ice with the wavanglet method is thus only slightly disturbed by the atmosphere contribution.

3) Grain Size Effect: Two ternary diagrams are calculated, one with a water ice grain size of $100 \mu \mathrm{m}$ (Fig. 6), a value 


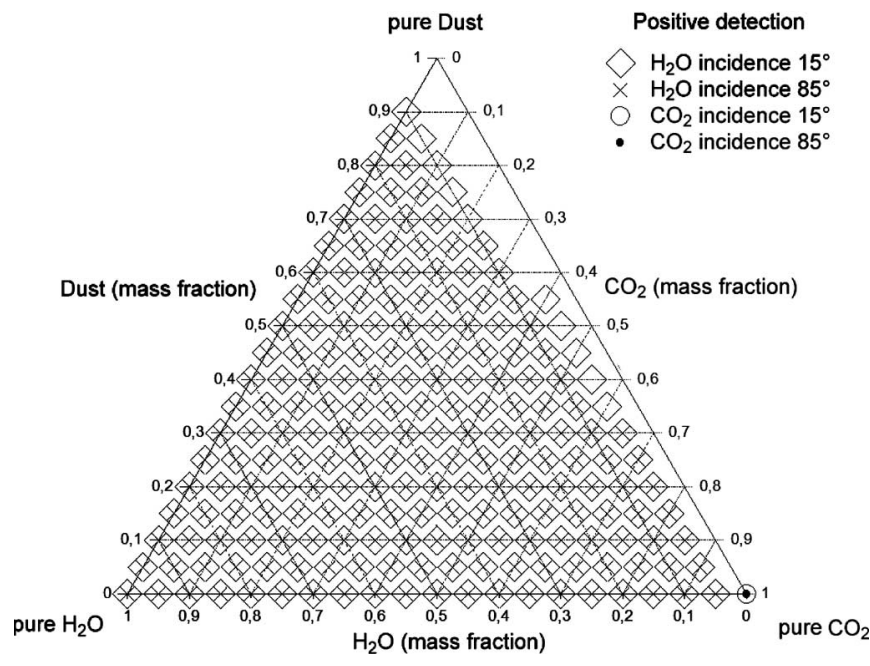

Fig. 6. Detection limits for $\mathrm{H}_{2} \mathrm{O}$ and $\mathrm{CO}_{2}$ for physical parameters of the typical surface (see Table VIII) of the permanent south polar cap. The atmosphere is typical for these conditions. The type of mixing is granular. Each symbol corresponds to positive detection of $\mathrm{H}_{2} \mathrm{O}$ ice (square and cross) and $\mathrm{CO}_{2}$ ice (circle and dot).

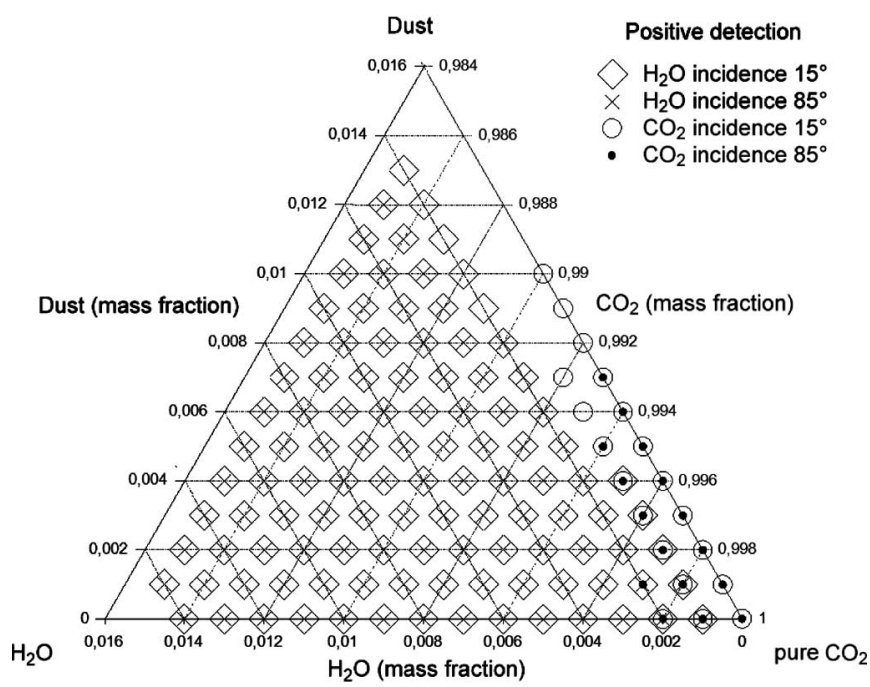

Fig. 7. Zoom on the lower right corner (pure $\mathrm{CO}_{2}$ ) of Fig. 6. Each symbol corresponds to positive detection of $\mathrm{H}_{2} \mathrm{O}$ ice (square and cross) and $\mathrm{CO}_{2}$ ice (circle and dot).

characteristics of the permanent south polar cap [10], [28], [29], and a second with a much higher value $(1000 \mu \mathrm{m}$, north polar cap [11], Fig. 9). Comparison of the two figures shows that the detection capacity strongly decreases as the grain size departs from the one used to generate the reference spectrum $(10 \mu \mathrm{m})$. This effect is due to the high nonlinear dependence of the $\mathrm{H}_{2} \mathrm{O}$ and $\mathrm{CO}_{2}$ spectral signatures with grain size that wavanglet cannot handle. In conclusion, for efficient detection, the real grain size on the site must not be too different from the value used for the endmember reference. Otherwise, we must modify or complete our reference base with more relevant spectra (simulated, measured, or observed) in order to improve the detection limit.

4) Incidence Angle: For a first approximation, the observation parameters (incidence, emergence, and azimuth angles) only influence the absolute band depth of the spectral features.

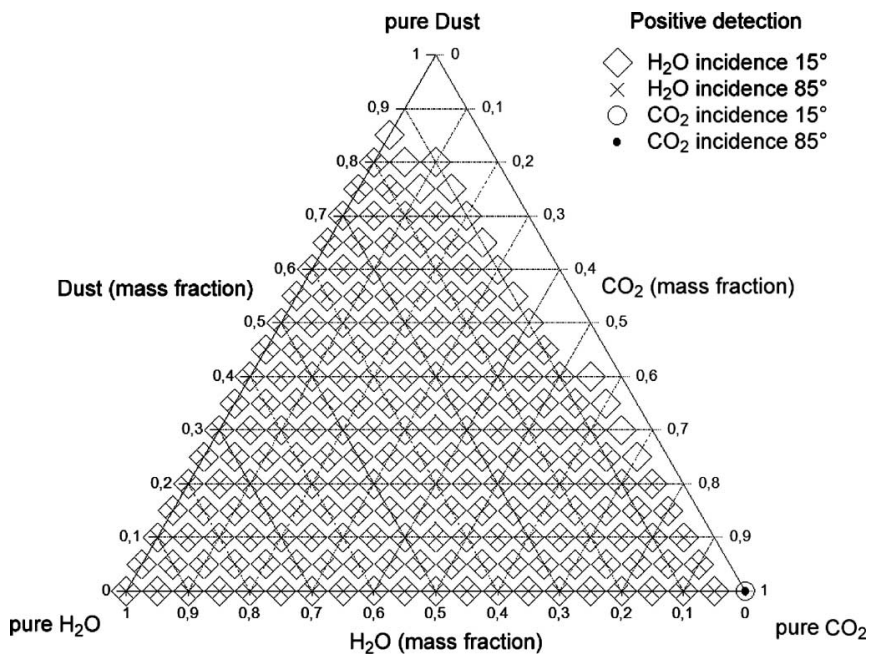

Fig. 8. Same conditions as in Fig. 6 except for a larger atmospheric path. Each symbol correspond to a positive detection of $\mathrm{H}_{2} \mathrm{O}$ ice (square and cross) and $\mathrm{CO}_{2}$ ice (circle and dot). Table VIII summarizes all parameters.

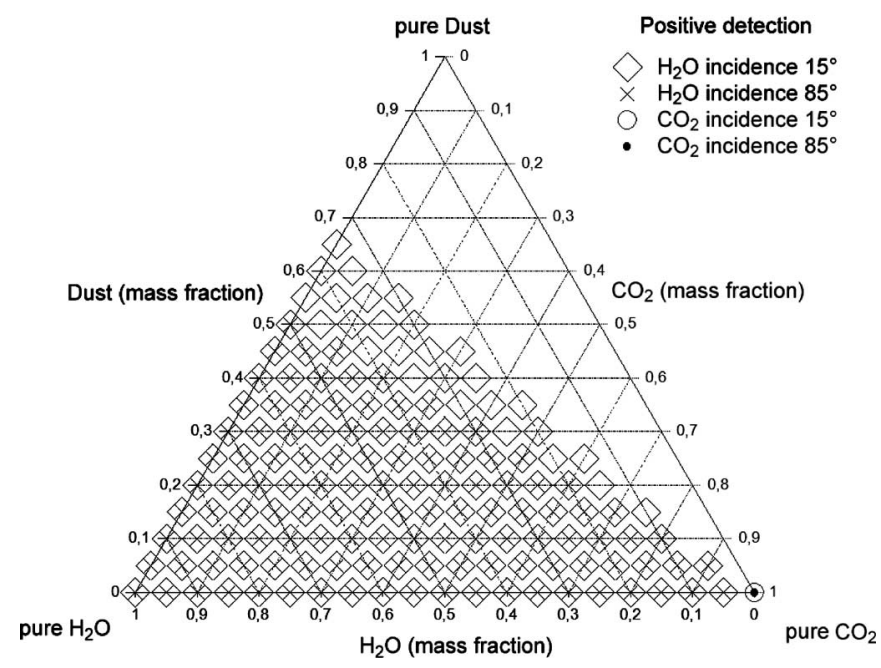

Fig. 9. Same conditions as in Fig. 6 except for a larger water grain size $(1000 \mu \mathrm{m})$ compared to the reference on the detection limits $(100 \mu \mathrm{m})$. Each symbol corresponds to positive detection of $\mathrm{H}_{2} \mathrm{O}$ ice (square and cross) and $\mathrm{CO}_{2}$ ice (circle and dot). Table VIII summarizes all parameters.

Since the wavanglet classification method is mostly sensitive to relative band depth, it may be fairly independent with respect to the illumination and observation conditions. The effect of the incidence angle has been tested for two extreme values: $85^{\circ}$ (inner symbols) and $15^{\circ}$ (outer symbols) for all the previous parameter sets (Figs. 6-9). The relative positioning of the inner and outer symbols for $\mathrm{H}_{2} \mathrm{O}$ and $\mathrm{CO}_{2}$ in all figures shows a very slightly better detection limit for $15^{\circ}$ incidence. Other calculations on the emergence angle near the nadir support the same conclusion. The wavanglet method appears to be independent of the geometrical conditions of the observation.

\section{Discussion AND Conclusion}

We have conducted a comparative study between two generic methods - the BR method and SFF-and wavanglet, our wavelet-based procedure. For this, we have considered 
TABLE IX

COMPARISON OF BR, SFF, AND WAVANGLET METHODS

\begin{tabular}{|c|c|c|c|}
\hline & Band Ratio (BR_fix) & Spectral Feature Fitting & Wavanglet \\
\hline \hline $\begin{array}{l}\text { Overall accuracy } \\
\text { (mean for } \mathrm{H}_{2} \mathrm{O} \text { and } \mathrm{CO}_{2} \text { ) }\end{array}$ & $57 \%$ & $83 \%$ & $\mathbf{8 9 \%}$ \\
\hline Class separability & Low & Overlap & High \\
\hline Large end-member dataset & Difficult & Possible & Possible \\
\hline Mass processing & Yes (No for BR_opt) & - & Yes \\
\hline Calculation time (s) & $\mathbf{1 1}$ & 41 & 54 \\
\hline Noise dependence & High and localized & - & Low \\
\hline
\end{tabular}

five criteria pertaining to automatic compound detection and classification of spectra: overall accuracy, separability between classes, multiple endmembers and possible overlapping signatures, mass processing feasibility, and calculation time. Table IX shows that only wavanglet possesses all the desired properties. Furthermore, it has the highest overall accuracy, outperforming SFF for the $\mathrm{CO}_{2}$ terrains. The BR_fix method usually misclassified a large fraction of the OMEGA images, and unfortunately BR_opt is not suitable for mass processing of complete datasets because a unique single detection threshold cannot be determined and applied to all OMEGA images. Also, BR is potentially very sensitive to noise for some spectels. Furthermore, wavanglet has two interesting properties for classifying our dataset: a reasonable calculation time and possible multidetections with a large endmember dataset. We demonstrated that the wavanglet method is able to discriminate between various spectral patterns that sometimes differ insufficiently and strongly overlap. We have also evaluated the sensitivity of wavanglet for the detection of $\mathrm{H}_{2} \mathrm{O}$ and $\mathrm{CO}_{2}$ ices as a function of certain physical as well as geometrical parameters using synthetic data. Thanks to the selection of medium and small-scale wavelets, the sensitivity of wavanglet is not hampered by variations of the incidence and emergence angles or by noise. Furthermore, the presence of atmospheric $\mathrm{CO}_{2}$ bands has a minor influence on detection. On the other hand, the nonlinear effect of grain size variability on the spectral signatures is difficult to handle with only one reference spectrum per material. To improve detectability over a wide range of surface textures, we propose selection of one endmember per terrain and per characteristic grain size in the reference base.

\section{ACKNOWLEDGMENT}

The authors would like to thank the two anonymous reviewers for interesting remarks. The authors would also like to thank the OMEGA team at Institut d'Astrophysique Spatiale for support with sequencing and data downloading activities, and also to M. Lonjaret, F. Madec, and T. Questier for usefull discussions.

\section{REFERENCES}

[1] J.-P. Bibring, A. Soufflot, M. Berthé, Y. Langevin, B. Gondet, P. Drossart, M. Bouyé, M. Combes, P. Puget, A. Semery, G. Bellucci, V. Formisano, V. Moroz, V. Kottsov, G. Bonello, S. Erard, O. Forni, A. Gendrin, N. Manaud, F. Poulet, G. Poulleau, T. Encrenaz, T. Fouchet, R. Melchiori, F. Altieri, N. Ignatiev, D. Titov, L. Zasova, A. Coradini, F. Capacionni, P. Cerroni, S. Fonti, N. Mangold, P. Pinet, B. Schmitt, C. Sotin, E. Hauber, H. Hoffmann, R. Jaumann, U. Keller, R. Arvidson, J. Mustard, and F. Forget, "OMEGA: Observatoire pour la Minéralogie, l'Eau, les Glaces et l'Activité," in Mars Express: The Scientific Payload. Noordwijk, Netherlands: ESA SP-1240, Aug. 2004, pp. 37-49.
[2] F. A. Kruse, A. B. Lefkoff, J. W. Boardman, K. B. Heidebrecht, A. T. Shapiro, P. J. Barloon, and A. F. H. Goetz, "The spectral image processing system (SIPS) - Interactive visualization and analysis of imaging spectrometer data," Remote Sens. Environ., vol. 44, no. 2/3, pp. 145-163, May/Jun. 1993.

[3] N. S. Subotic, J. D. Gorman, and B. J. Thelen, "Wavelet techniques for band selection and material classification from hyperspectral data," in Proc. SPIE_Imaging Spectrometry, M. R. Descour, J. M. Mooney, D. L. Perry, and L. R. Illing, Eds., Jun. 1995, vol. 2480, pp. 426-434.

[4] L. M. Bruce, and J. Li, "Fast wavelet-based algorithms for multiresolutional decomposition and feature extraction of hyperspectral signatures," in Proc. SPIE-Algorithms for Multispectral and Hyperspectral Imagery V, S. S. Shen and M. R. Descour, Eds., Jul. 1999, vol. 3717, pp. 72-81.

[5] L. M. Bruce, C. H. Koger, and J. Li, "Dimensionality reduction of hyperspectral data using discrete wavelet transform feature extraction," IEEE Trans. Geosci. Remote Sens., vol. 40, no. 10, pp. 2331-2338, Oct. 2002.

[6] S. Kaewpijit, J. Le Moigne, and T. El-Ghazawi, "Automatic reduction of hyperspectral imagery using wavelet spectral analysis," IEEE Trans. Geosci. Remote Sens., vol. 41, no. 4, pp. 863-871, Apr. 2003.

[7] C. H. Koger, L. M. Bruce, D. R. Shaw, and K. N. Reddy, "Wavelet analysis of hyperspectral reflectance data for detecting pitted morning glory (ipomoea lacunosa) in soybean (glycine max)," Remote Sens. Environ., vol. 86, no. 1, pp. 108-119, Jun. 2003.

[8] H. Wu, G. Kuang, and W. Yu, "Unsupervised classification method for hyperspectral image combining PCA and Gaussian mixture model," in Proc. 3rd Int. Symp. Multispectr. Image Process. and Pattern Recognit., H. Lu and T. Zhang, Eds., Sep. 2003, vol. 5286, pp. 729-734.

[9] J. E. Pinzon, S. L. Ustin, C. M. Castaneda, J. F. Pierce, and L. A. Costick, "Robust spatial and spectral feature extraction for multispectral and hyperspectral imagery," in Proc. SPIE_Algorithms for Multispectral and Hyperspectral Imagery IV, S. S. Shen and M. R. Descou, Eds., Jul. 1998, vol. 3372, pp. 199-210.

[10] J. Bibring, Y. Langevin, F. Poulet, A. Gendrin, B. Gondet, M. Berthé, A. Soufflot, P. Drossart, M. Combes, G. Bellucci, V. Moroz, N. Mangold, and B. Schmitt, "Perennial water ice identified in the south polar cap of Mars," Nature, vol. 428, no. 6983, pp. 627-630, Apr. 2004.

[11] Y. Langevin, F. Poulet, J.-P. Bibring, B. Schmitt, S. Douté, and B. Gondet, "Summer evolution of the north polar cap of Mars as observed by OMEGA/Mars express," Science, vol. 307, no. 5715, pp. 1581-1584, Mar. 2005.

[12] R. N. Clark, A. J. Gallagher, and G. A. Swayze, "Material absorption band depth mapping of imaging spectrometer data using the complete band shape least squares algorithm simultaneously fit to multiple spectral features from multiple materials," in Proc. 3rd AVIRIS Workshop, 1990, pp. 176-186.

[13] S. Mallat, A Wavelet Tour of Signal Processing. New York: Academic, 1999, ch. 4, pp. 107-121.

[14] W. H. Press, B. P. Flannery, S. A. Teukolsky, and W. T. Vetterling, Numerical Recipes in Fortran 77: The Art of Scientific Computing. Cambridge, U.K.: Cambridge Univ. Press, 1986-1992, ch. 13.10, pp. 584-599.

[15] Research Systems, Inc., "Spectral tools," in ENVI User's Guide, Research Systems, Boulder, CO, 2000, ch. 9, pp. 615-686.

[16] J. Boardman, F. A. Kruse, and R. O. Green, "Mapping target signatures via partial unmixing of AVIRIS data: In summaries," in Proc. 5th JPL Airborne Earth Sci. Workshop, JPL Publication 95-1, Mar. 1995, vol. 1, pp. 23-26.

[17] A. Green, M. Berman, P. Switzer, and M. Craig, "A transformation for ordering multispectral data in terms of image quality with implications for noise removal," IEEE Trans. Geosci. Remote Sens., vol. 26, no. 1, pp. 65-74, Jan. 1988.

[18] S. Douté and B. Schmitt, "A multilayer bidirectional reflectance model for the analysis of planetary surface hyperspectral images at visible and near-infrared wavelengths," J. Geophys. Res., vol. 103, no. 12, pp. $31367-$ 31 390, Dec. 1998. 
[19] L. Renzullo, A. Blanchfield, and K. Powell, "A method of wavelength selection and spectral discrimination of hyperspectral reflectance spectrometry," IEEE Trans. Geosci. Remote Sens., vol. 44, no. 7, pp. 19861994, Jul. 2006.

[20] C.-I Chang and S. Wang, "Constrained band selection for hyperspectral imagery," IEEE Trans. Geosci. Remote Sens., vol. 44, no. 6, pp. 15751585, Jun. 2006.

[21] J. Li, "Wavelet-based feature extraction for improved endmember abundance estimation in linear unmixing of hyperspectral signals," IEEE Trans. Geosci. Remote Sens., vol. 42, no. 3, pp. 644-649, Mar. 2004.

[22] S. Liangrocapart and M. Petrou, "Feasibility study on the use of nonlinear spectral unmixing," in Proc. SPIE-Image and Signal Processing for Remote Sensing V, S. B. Serpico, Ed., Dec. 1999, vol. 3871, pp. 159-168.

[23] R. R. Coifman and M. V. Wickerhauser, "Entropy-based algorithms for best basis selection," IEEE Trans. Inf. Theory, vol. 38, no. 2, pp. 713718, Mar. 1992.

[24] N. Saito and R. R. Coifman, "Local discriminant bases," in Proc. SPIE-Wavelet Applications in Signal and Image Processing II, A. F. Laine and M. A. Unser, Eds., Oct. 1994, vol. 2303, pp. 2-14.

[25] E. Quirico and B. Schmitt, "Near-infrared spectroscopy of simple hydrocarbons and carbon oxides diluted in solid $\mathrm{N}_{2}$ and as pure ices: Implications for Triton and Pluto," Icarus, vol. 127, no. 2, pp. 354-378, Jun. 1997.

[26] W. M. Grundy and B. Schmitt, "The temperature-dependent near-infrared absorption spectrum of hexagonal $\mathrm{H}_{2} \mathrm{O}$ ice," J. Geophys. Res., vol. 103, no. E11, pp. 25 809-25 822, Nov. 1998.

[27] B. Schmitt, E. Quirico, F. Trotta, and W. M. Grundy, "Optical properties of ices from UV to infrared," in Proc. ASSL-Solar System Ices, 1998, vol. 227, pp. 199-240.

[28] S. Douté, B. Schmitt, Y. Langevin, J.-P. Bibring, F. Altieri, G. Bellucci, and B. Gondet, "South pole of Mars: Nature and composition of the icy terrains from Mars express OMEGA observations," Planet. Space Sci., vol. 55, no. 1-2, pp. 113-133, Jan. 2007.

[29] D. A. Glenar, G. Hansen, G. Bjoraker, M. Smith, J. Pearl, and D. Blaney, , "Bright-region radiative properties within the Mars south polar cap $(\mathrm{Ls}=231)$ from near-infrared spectroscopic imaging," Icarus, vol. 174 , no. 2 , pp. $600-603$, Apr. 2005.

[30] S. A. Clough, M. W. Shephard, E. J. Mlawer, J. S. Delamere, M. J. Iacono, K. Cady-Pereira, S. Boukabara, and P. D. Brown, "Atmospheric radiative transfer modeling: a summary of the AER codes," J. Quant. Spectrosc. Radiat. Transf., vol. 91, no. 2, pp. 233-244, Mar. 2005.

[31] R. Congalton and K. Green, Assessing the Accuracy of Remotely Sensed Data: Principles and Practices, J. Lyon, Ed. New York: Lewis, 1999.

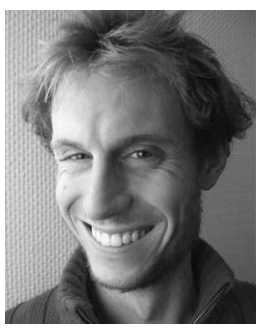

Frédéric Schmidt received the two-years degree in physics from the Université Louis Pasteur, Strasbourg, France, in 2001, the B.S. degree in earth science from the Ecole Normale Supérieure, Lyon, France, in 2003, and the M.S. degree in earth science from the Université Joseph Fourier (UJF), Grenoble, France, in 2004. He is currently working toward the Ph.D. degree at the Laboratoire de Planétologie de Grenoble (Centre National de la Recherche Scientifique-UJF), Grenoble.

His research interests are analysis of hyperspectral data, statistics in geophysical fields, ices on planet Mars, and Martian climate. In particular, he is working on hyperspectral images produced by the OMEGA imaging spectrometer (MEX, ESA).

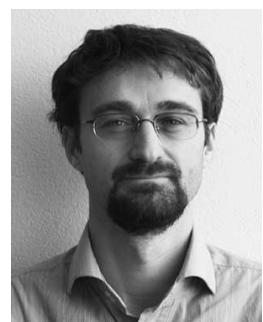

Sylvain Douté received the B.S. and M.S. degrees from the Université Joseph Fourier (UJF), Grenoble, France, in 1991 and 1994, respectively, and the Ph.D. degree from the Université Denis Diderot, Paris, France, after working three years at the Laboratoire de Glaciologie et Géophysique de l'Environnement, Grenoble.

He held a postdoctoral position for two years at the Institute of Geophysics and Planetary Physics (University of California, Los Angeles) to analyze images of the near-infrared mapping spectrometer (Galileo, NASA). He is currently a Researcher with the Laboratoire de Planétologie de Grenoble (CNRS-UJF), Grenoble. His research interests include remote sensing of planetary surfaces by hyperspectral imaging, theoretical as well as experimental study of solar light radiative transfer in geophysical media. In particular, he is working on images produced by the OMEGA imaging spectrometer (MEX, ESA).

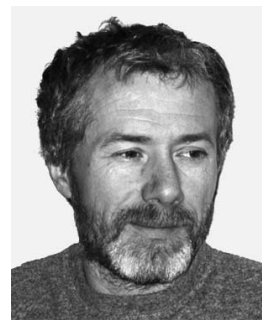

Bernard Schmitt received the Ingenieur degree (material sciences) from the Institut National Polytechnique de Grenoble, Grenoble, France, in 1983, and the Ph.D. degree in physics (material sciences) from the University J. Fourier, Grenoble, in 1986.

From 1988 to 1999, he was affiliated with the Laboratoire de Glaciologie et Géophysique de l'Environnement (CNRS) and contributed to create in 1999 the Laboratoire de Planétologie de Grenoble (CNRS-University J. Fourier) where he is currently Research Director of a group working on solidmatter spectroscopy and remote sensing of solar system surfaces. His research interests include visible to infrared laboratory spectroscopy and radiometry of ices and molecular solids, and hyperspectral remote sensing of planetary surfaces. He is Coinvestigator of several imaging spectrometers onboard space missions (OMEGA/MEX, DISR/Casini-Huygens, VIRTIS/Rosetta, etc.). 